\title{
Microporation is a valuable transfection method for efficient gene delivery into human umbilical cord blood-derived mesenchymal stem cells
}

\author{
Jung Yeon Lim¹', Sun Hwa Park¹, Chang Hyun Jeong1', Ji Hyeon Oh1', Seong Muk Kim¹, Chung Hun Ryu1, \\ Soon A Park1, Jae Geun Ahn³, Wonil Oh4', Sin-Soo Jeun*1,2 and Jong Wook Chang ${ }^{4}$
}

\begin{abstract}
Background: Mesenchymal stem cells (MSCs) are an attractive source of adult stem cells for therapeutic application in clinical study. Genetic modification of MSCs with beneficial genes makes them more effective for therapeutic use. However, it is difficult to transduce genes into MSCs by common transfection methods, especially nonviral methods. In this study, we applied microporation technology as a novel electroporation technique to introduce enhanced green fluorescent protein (EGFP) and brain-derived neurotropfic factor (BDNF) plasmid DNA into human umbilical cord blood-derived MSCs (hUCB-MSCs) with significant efficiency, and investigated the stem cell potentiality of engineered MSCs through their phenotypes, proliferative capacity, ability to differentiate into multiple lineages, and migration ability towards malignant glioma cells.
\end{abstract}

Results: Using microporation with EGFP as a reporter gene, hUCB-MSCs were transfected with higher efficiency (83\%) and only minimal cell damage than when conventional liposome-based reagent $(<20 \%)$ or established electroporation methods were used (30-40\%). More importantly, microporation did not affect the immunophenotype of hUCB-MSCs, their proliferation activity, ability to differentiate into mesodermal and ectodermal lineages, or migration ability towards cancer cells. In addition, the BDNF gene could be successfully transfected into hUCB-MSCs, and BDNF expression remained fairly constant for the first 2 weeks in vitro and in vivo. Moreover, microporation of BDNF gene into hUCBMSCs promoted their in vitro differentiation into neural cells.

Conclusion: Taken together, the present data demonstrates the value of microporation as an efficient means of transfection of MSCs without changing their multiple properties. Gene delivery by microporation may enhance the feasibility of transgenic stem cell therapy.

\section{Background}

Stem cells are highly attractive and valuable candidates for biomedical applications including the development of cell and gene therapy. Of the various stem cells, mesenchymal stem cells (MSCs) show a particular potential for clinical use because of their high proliferative capacity, ability to differentiate into multiple lineages [1-3], and ability to migrate into injured organs $[4,5]$ and cancers $[6,7]$. Moreover, MSCs are not immunogenic, and so do

* Correspondence: ssjeun@catholic.ac.kr

1 Department of Biomedical Science, College of Medicine, The Catholic University of Korea, Seoul, Korea

+ Contributed equally

Full list of author information is available at the end of the article not elicit a proliferative response of allogeneic lymphocytes in vitro [8]. Therefore, MSCs have become a major focus of research for potential therapeutic applications for various diseases.

In recent years, efforts have been made to improve the therapeutic efficacy of MSCs through combination approaches using MSCs and genes. Additionally, a new therapeutic strategy has been developed that uses MSCs for the targeted delivery and local production of biologic agents in tumors [6,9]. Viruses are commonly used as vehicles to deliver transgenes into stem cells that can be available to effectively infect dividing or nondividing cells. Integrating virus, including retrovirus or lentivirus, 
can insert their viral DNA into the host genomic DNA, which allows for stable genetic modification for the life of the host cells. So, they are very efficient for long-term gene expression [10-12]. Alternatively, nonintegrating viruses, including adenovirus or herpes saimirii virus, are preferentially used to obtain the expression of a therapeutic gene for a short time, although these viruses are less efficient at transferring genes into cells [13]. Overall, virus systems permit efficient gene delivery into cells; however, they have safety concerns that are critical when considering clinical applications [14]. Furthermore, their use sometimes causes significant changes in the characteristics of genetically modified cells [15].

To overcome these problems, nonviral methods are receiving increasing attention because they have several potential advantages over recombinant viruses. They are noninfectious, relatively nonimmunogenic, have low acute toxicity, can accommodate large DNA plasmids, and can be produced simply on a large scale [16]. There are various types of nonviral systems used for gene transfer, such as the liposome-based method [17], electroporation [18], and calcium phosphate techniques [19]. Electroporation, which permeabilizes the cell membrane by an electric pulse, has been widely used [20-22]. However, these methods are limited by their low gene transfer efficiency compared with viruses and their transient gene expression [16]. In addition, high cell mortality is still a problem in electroporation. Low levels of gene transfer continue to be a major obstacle in the use of nonviral systems because gene delivery and efficient gene transfer are prerequisites for the development of MSC therapy using various beneficial genes in clinical trials.

In contrast to conventional electroporation methods, microporation is a unique electroporation technology that uses a pipette tip as an electroporation space and a capillary type of electric chamber instead of a cuvette, which counteracts the harmful effects of cuvette-based electroporation gene transfer techniques such as $\mathrm{pH}$ variation, increasing in temperature, and metal ion generation.

The present study reports a nonviral, high-efficiency method of transfecting human umbilical cord bloodderived MSCs (hUCB-MSCs) using the new electroporation-based gene transfer technique of microporation. Microporation was utilized to introduce enhanced green fluorescent protein (EGFP) as a reporter gene and a plasmid expressing the gene of interest, brain-derived neurotrophic factor (BDNF), into hUCB-MSCs with significant efficiency, and investigated the stem cell potentiality of engineered hUCB-MSCs through their phenotypes, proliferative capacity, ability to differentiate into multiple lineages, and migration ability towards malignant glioma cells.

\section{Results \\ Microporation of hUCB-MSCs Induces High Transient Transfection Efficiency}

To investigate the gene transfection efficiency of the microporation technique, hUCB-MSCs were transfected with EGFP-N1 plasmid (pEGFP-N1) by various transfection methods including liposome-based reagent, established electroporations, and microporation. For electroporation, $2 \times 10^{5}$ cells were resuspended in phosphate buffered saline (PBS) or resuspension buffer, mixed with $2 \mu \mathrm{g}$ DNA and electroporated as described previously $[23,24]$. For microporation, $2 \mu \mathrm{g}$ DNA was mixed with resuspension buffer. Then, $2 \times 10^{5}$ cells were electroporated with a preoptimized square pulse condition ( $1600 \mathrm{~V}, 20 \mathrm{~ms}, 1$ pulse). Figure 1A showed the brightfield and green fluorescence images of cells. In addition, the percentage of EGFP-positive cells $24 \mathrm{hr}$ after transfection was determined by fluorescence-activated cell sorting (FACS) analysis (Figure 1B). The percentage of EGFPpositive cells was $30 \%-50 \%$ for the established electroporators, Nucleofector, ECM 830 or MicroPulser ${ }^{\text {twx }}$. By comparison, the MicroPorator ${ }^{\mathrm{mm}}$ microporation device achieved a transfection efficiency of $83 \%$ under the conditions tested. In addition, microporation-based transfection of hUCB-MSCs showed a higher cell survival rate compared with other systems (Figure 1C). Although Gene Porter2-based transfection of hUCB-MSCs demonstrated a high cell survival rate, EGFP expression was too low $(<20 \%)$.

We further examined the survival of microporated cells during 15 days culture of actively dividing cells. Cell viability was about $80 \% 2$ days after microporation, and viability was sustained for more than 15 days (Figure 2A). To increase the application performance of microporation, transfection efficacies were examined in different hUCBMSCs from different donors. Similar transfection efficiencies were obtained (Figure 2B).

To assess the stability of the microporation-based transfection, the time course and the level of EGFP expression were determined over a period of 3 weeks under conditions of restrained cell division (a-minimal essential medium (MEM) containing $2 \%$ fetal bovine serum (FBS) and no passaging). Fluorescence images and FACS analysis at different time points after transfection revealed that EGFP expression was sustained at high levels $(>60 \%)$ for the first 10 days and began to decline after 2 weeks (Figure 3A). We also examined the EGFP expression level in actively dividing cells under standard culture conditions (a-MEM containing 10\% FBS and subculturing at $90 \%$ confluency). Similarly, EGFP expression was sustained at high levels $(>60 \%)$ for the first 10 days and began to decline after 15 days (Figure 3B). By comparison, EGFP expression was decreased markedly 10 days 
A

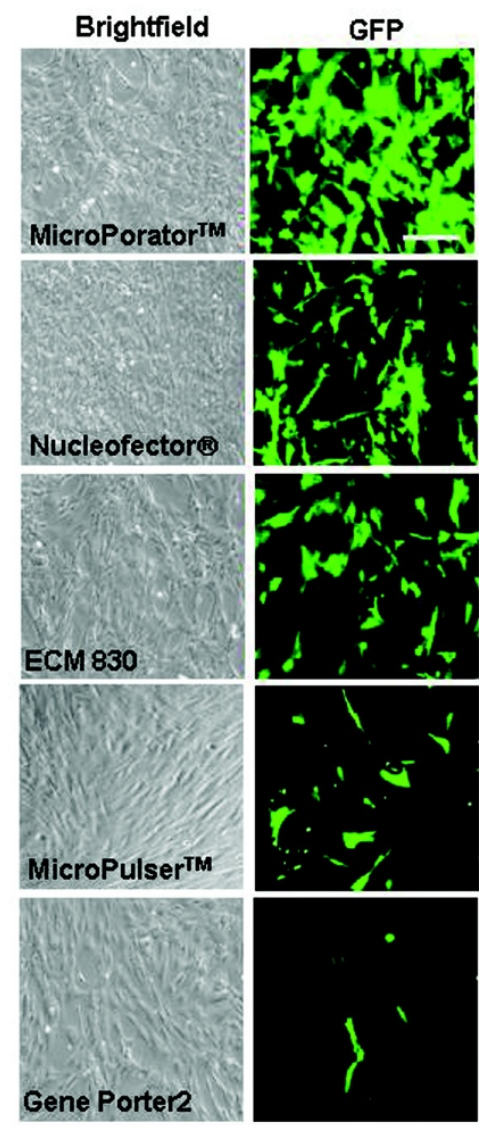

B

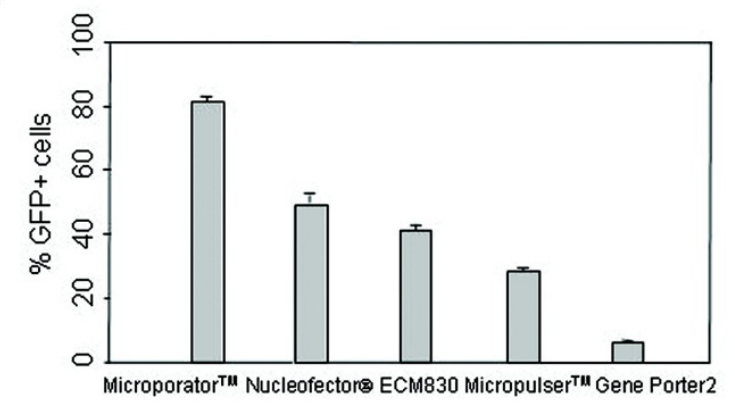

C

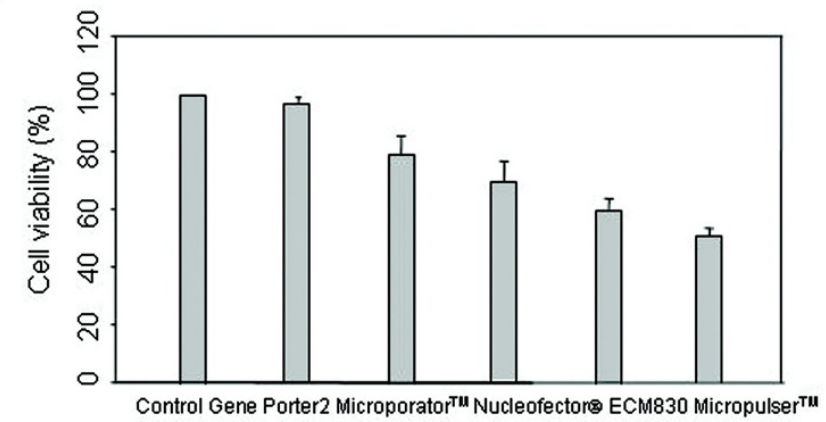

Figure 1 Transfection efficiency of hUCB-MSCs. (A) pEGFP-N1 vector was transfected into hUCB-MSCs by the established electroporators Nucleofector ${ }^{\circ}$, ECM 830, MicroPulser ${ }^{\mathrm{TM}}$, and a novel electroporator, MicroPorator ${ }^{\mathrm{TM}}$, or by a liposome-based reagent, Gene Porter2. After 24 hr, EGFP expression was analyzed using phase contrast and fluorescence microscopy. (B) Transient expression of EGFP was assessed by FACS analysis. (C) After transfection, cells were cultured for additional $48 \mathrm{hr}$ and analyzed for viability by the MTS assay. The data are expressed as the mean \pm SEM; $\mathrm{n}=3$. Scale bar $=200 \mu \mathrm{m}$.

after nucleofection-based transfection, and its expression level was weak (Figure 4). These results indicate that transfection by microporation is an efficient way of obtaining successful gene delivery into MSCs.

\section{Multiple Stem Cell Traits of EGFP-expressing hUCB-MSCs by Microporation}

To investigate the stem cell potentiality of hUCB-MSCs according to transfection by Microporatot ${ }^{\mathrm{Th}}$, cell proliferative capacity was evaluated by a trypan blue exclusion assay. Both non-transfected hUCB-MSCs and hUCBMSCs transfected with pEGFP-N1 grew in a similar fashion in a time-dependent manner (Figure 5A). The in vitro culture of cells appeared to be unaffected by transfection using the microporation.

To further study stem cell potentiality, the expression of MSC-related cell surface antigens was evaluated by flow cytometry (Figure 5B). Like hUCB-MSCs, transfected hUCB-MSCs were strongly positive for the CD29, CD44,
CD73, and CD90 markers. However, both cells stained negatively for HLA-class II (HLA-DR) and the hematopoietic marker CD34.

The ability to differentiate into a variety of cell types is an important characteristic of MSCs [2,3]. To evaluate the influence of microporation on multilineage differentiation of hUCB-MSCs, hUCB-MSCs and hUCB-MSCs transfected with pEGFP-N1 by microporation were induced into osteogenic and adipogenic differentiation. Both cells showed similar differentiation into the osteogenic and adipogenic lineages when grown in culture with the appropriate differentiation medium. Osteogenic differentiation was determined by von Kossa staining (Figure 5C). Adipogenic differentiation was apparent in the cells by cellular accumulation of lipid-rich vacuoles that were stained with Oil Red O (Figure 5D). EGFP expression was maintained in hUCB-MSCs during the osteoinductive conditions (Figure 5E). 
A
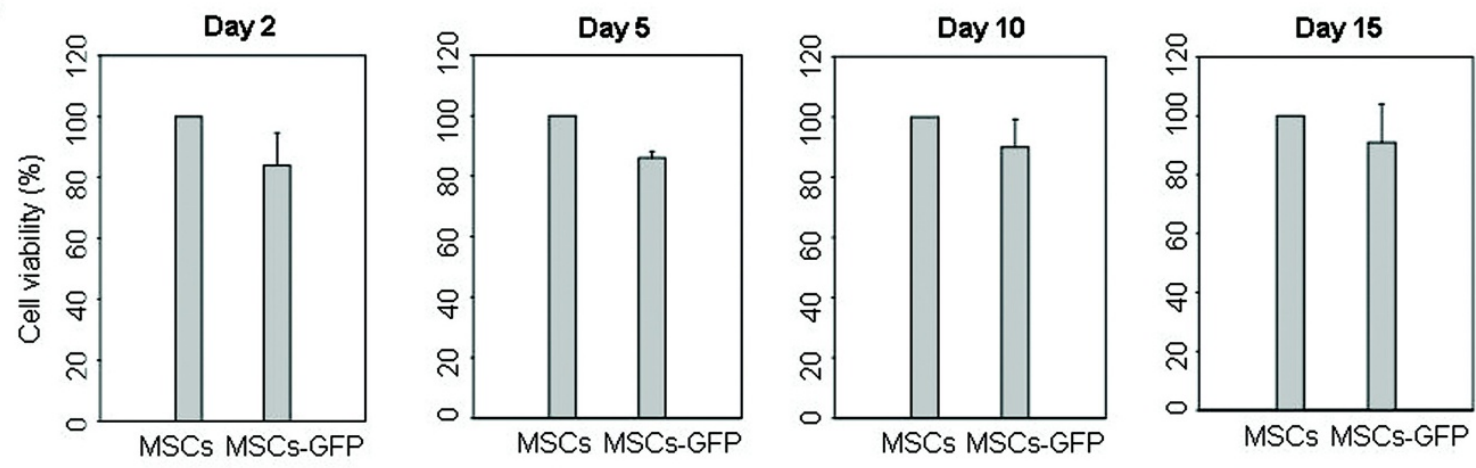

\section{B}
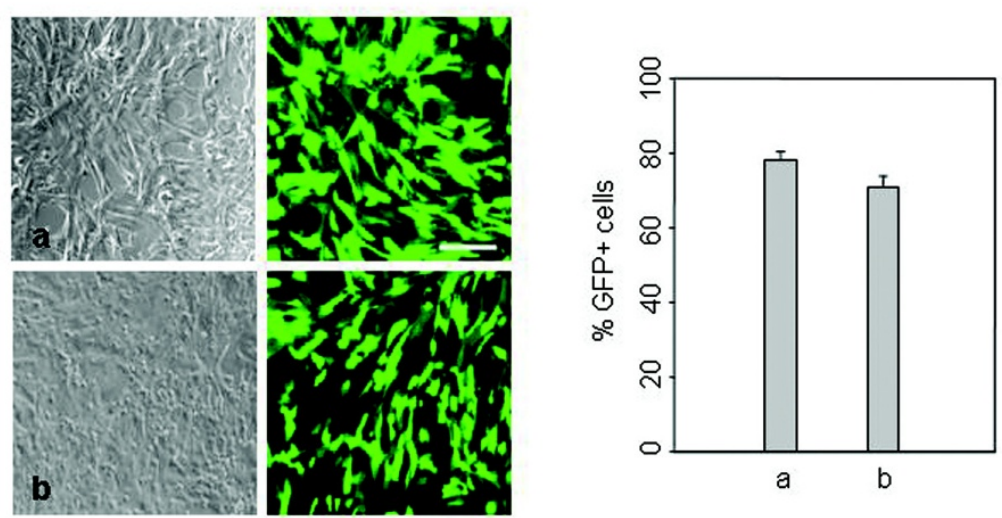

Figure 2 Transfection efficiency of hUCB-MSCs from different donors. (A) After microporation, cells survival was analyzed by the MTS assay at different time points under standard culture conditions (a-MEM containing 10\% FBS and subculturing at 90\% confluency). (B) pEGFP-N1 vector was transfected into different hUCB-MSCs from different donors by microporation. After $24 \mathrm{hr}$, the expression of EGFP was analyzed using phase contrast and fluorescence microscopy. Transient expression of EGFP was assessed by FACS analysis. The data are expressed as the mean \pm SEM; $n=3$. Scale bar $=200 \mu \mathrm{m}$.

Migration capacity towards cancer cells is an important characteristic of MSCs. Recent studies have indicated that MSCs exhibit potent tropism for malignant glioma cells $[7,9]$. To test this in hUCB-MSCs transfected with pEGFP-N1 by microporation, in vitro migration assays using Transwell plates were conducted. Conditioned medium (CM) from normal human astrocytes was used as a control to mimic the normal brain milieu. Only a few cells migrated toward serum-free medium (SFM) and $\mathrm{CM}$ from normal human astrocytes, whereas the migration of cells was significantly stimulated by $\mathrm{CM}$ from the human glioma cell line U-87MG compared with CM from astrocytes. We also confirmed that transfected hUCB-MSCs migrated towards CM from U-87MG cells in a similar pattern to hUCB-MSCs (Figure 6). In addition, the expression of EGFP was observed in migrated hUCB-MSCs. Thus, the migratory ability of hUCB-MSCs was not affected by microporation.

\section{Characterization of BDNF-expressing hUCB-MSCs by Microporation}

To investigate the potential for application of microporated hUCB-MSCs for cell therapy, the gene of interest harbored in a BDNF expression plasmid (pEGFP-BDNF) was transfected into hUCB-MSCs and the transgene expression and its effects for promoting in vitro differentiation of hUCB-MSCs into neural cells were examined. BDNF regulates the survival, development, and function of neurons, and is important for differentiation of neural precursor cells $[25,26]$. Our previous report demonstrated that BDNF stimulates in vitro differentiation of MSCs into neural cells [27]. In addition, MSCs genetically modified with the BDNF gene effectively promote axonal regeneration in transected adult rat spinal cord [28]. Therefore, if hUCB-MSCs could be successfully modified with BDNF gene by microporation, it would be a valuable cell source for the treatment of neurological disease. 
A
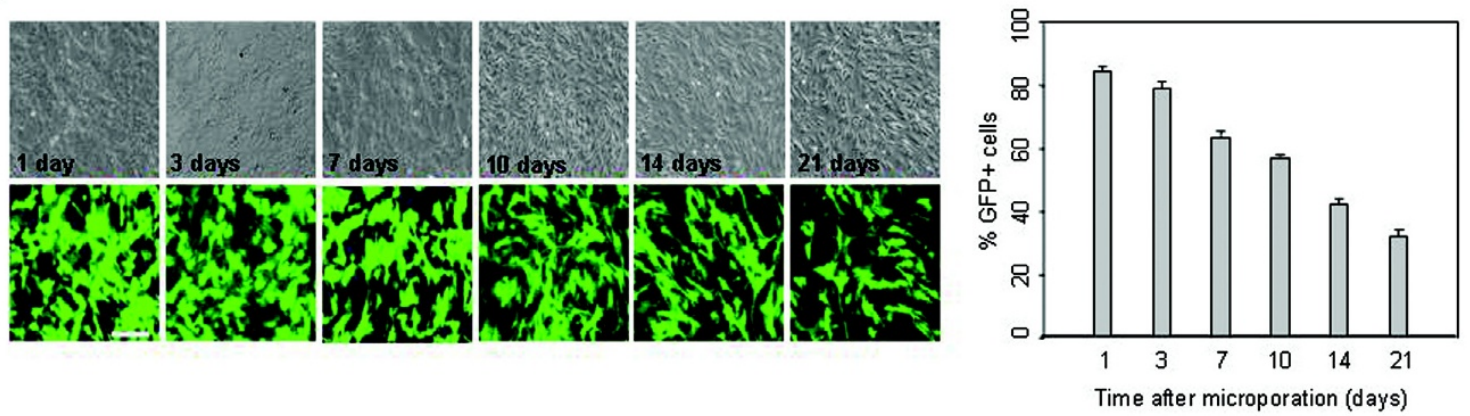

B
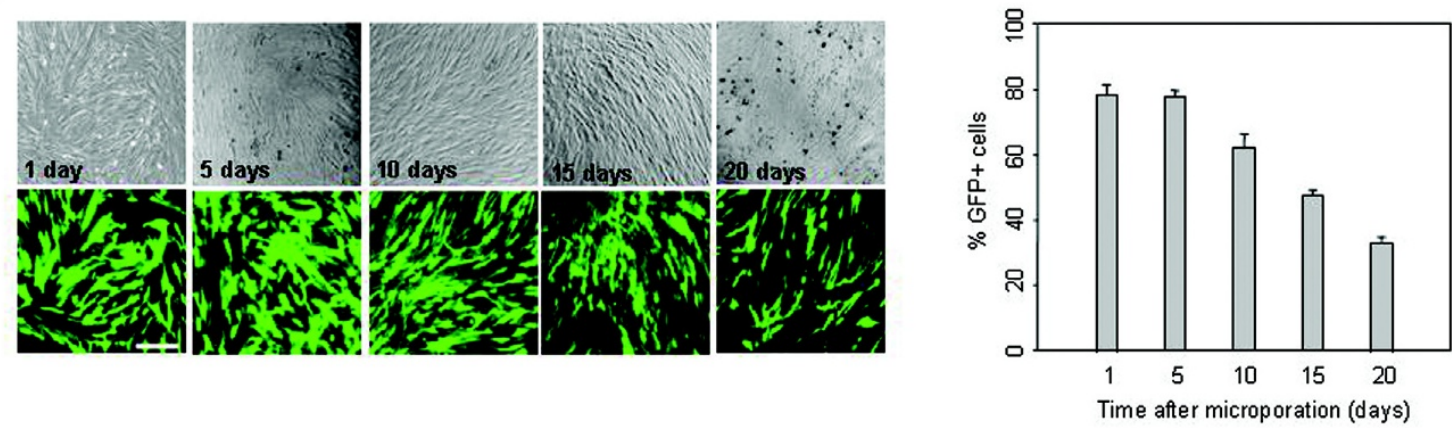

Figure 3 Time-course analysis of EGFP expression in microporated hUCB-MSCs. (A) pEGFP-N1 (2 $\mu \mathrm{g}$ ) was transfected into hUCB-MSCs by microporation. The expression of EGFP was analyzed using phase contrast and fluorescence microscopy at different time points after transfection. Persistence of EGFP expression in hUCB-MSCs was analyzed by FACS analysis under conditions of restrained cell division (a-MEM containing $2 \%$ FBS and no passaging). EGFP expression was assessed at different time points after transfection. (B) Persistence of EGFP expression in microporated hUCBMSCs was analyzed by FACS analysis under standard culture conditions. EGFP expression was assessed at different time points after transfection. The data are expressed as the mean \pm SEM; $n=3$. Scale bar $=200 \mu \mathrm{m}$.

Enzyme-linked immunosorbant assay (ELISA) analysis at different time points after transfection revealed that the concentration of secreted BDNF in vitro remained fairly constant for 14 days and then began to decline in transfected hUCB-MSCs (Figure 7A, left panel). Additionally, immunocytochemical analysis showed strong staining for BDNF in the transfected cells (Figure 7A, right panel). The levels of BDNF protein and its longevity in vivo were quantitatively measured. ELISA analysis on different days after transplantation of cells into rat brain revealed that the BDNF protein expressed in brain tissues was detectable on day 1 , peaked on day 7 , and persisted for two weeks. In control groups, expression levels of BDNF were low throughout the experimental period (Figure 7B, left panel). In addition, immunohistochemical analysis 3 days after transplantation showed strong staining for BDNF in the injection site or areas around the injection site of BDNF-expressing hUCB-MSCs (Figure 7B, right panel).

To investigate the effect of BDNF in promoting in vitro differentiation of hUCB-MSCs into neural cells, cell morphology was observed when hUCB-MSCs were treated with neural induction medium (NIM), with or without transfection of BDNF expression plasmid by microporation Non-transfected or transfected hUCB-MSCs were incubated overnight in preinduction medium containing bFGF. Next, they were transferred to NIM, and then maintained for up to 7 days. hUCB-MSCs transfected with BDNF expression plasmid progressively assumed neuronal morphological characteristics. The cytoplasm in the flat hUCB-MSCs retracted toward the nucleus, resembling a typical neuronal soma, and the process became thinner because of continuous shrinkage of the cell body. The morphological changes progressed to yield network-like structures and many long processes. However, these morphological changes were weak for the non-transfected hUCB-MSCs, and only a few cells showed pyramidal or spherical cell bodies with multiple processes (Figure 8A). In addition to their neural morphological differentiation, the effects of BDNF were investigated by Western blot analysis. In the proliferating medium, MSCs highly expressed the intermediate filament protein nestin. But, the expression of nestin decreased dramatically after induction. MSCs were nega- 

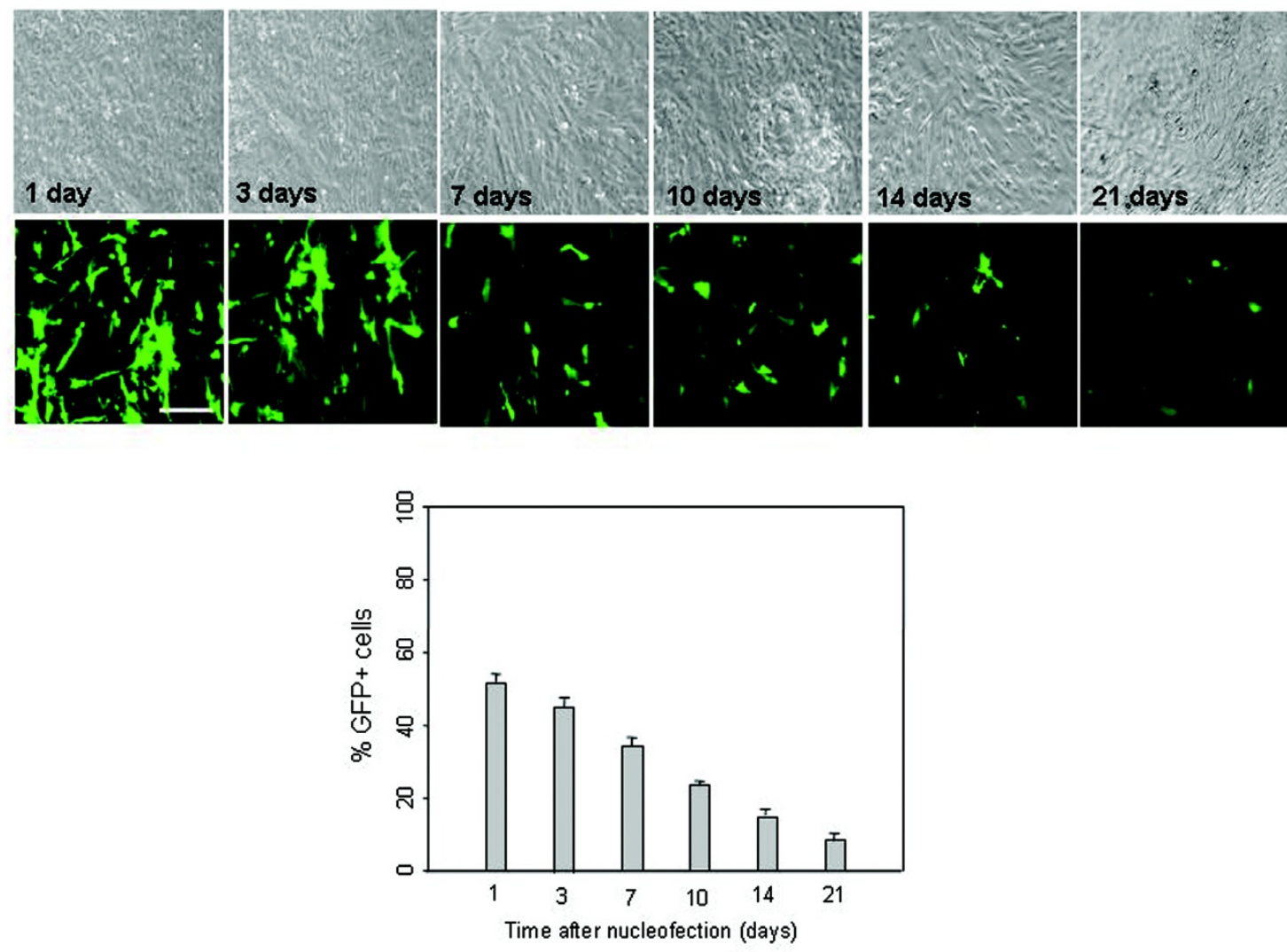

Figure 4 Time-course analysis of EGFP expression in nucleofected hUCB-MSCs. pEGFP-N1 $(2 \mu \mathrm{g})$ was transfected into hUCB-MSCs by nucleofection. The expression of EGFP was analyzed using phase contrast and fluorescence microscopy at different time points after transfection. Persistence of EGFP expression in hUCB-MSCS was analyzed by FACS analysis under conditions of restrained cell division (a-MEM containing $2 \%$ FBS and no passaging). EGFP expression was assessed at different time points after transfection. The data are expressed as the mean \pm SEM; $n=3$. Scale bar $=200 \mu m$.

tive for several neural proteins including the differentiating neuron marker Tuj-1, the mature neuron marker NeuN, GFAP typical of astrocytes, and the oligodendrocyte marker MBP. Conversely, expression of Tuj-1 and NeuN increased significantly after neurogenic stimulation in BDNF-transfected hUCB-MSCs. In addition, expression of GFAP and MBP increased after induction in transfected cells. However, these increases were marginal for those non-transfected cells treated with NIM (Figure 8B).

Next, the neural differentiation-related molecules in the hUCB-MSCs and BDNF-transfected hUCB-MSCs were examined after neural induction. Mitogen-activated protein kinases are expressed abundantly in the central nervous system, and extracellular signal-regulated kinases (ERKs) participate in various activity-dependent processes, including neuronal maturation, survival, and synaptic functions. The phosphorylation levels of Raf-1 and ERKs were dramatically increased in BDNF-transfected hUCB-MSCs at 7 days of induction (Figure 8C).

\section{Discussion}

MSCs therapy using therapeutic transgene holds the potential as a powerful means of disease treatment. For example, MSCs genetically modified with the BDNF gene effectively promote axonal regeneration in transected adult rat spinal cord [28] and also protect against injury in a cerebral ischemia model in adult rats [29]. MSCs genetically modified with bone morphogenic protein-2 (BMP-2) gene show good results in treating bone defects, and engineering MSCs with BMP-2 has recently been the focus for research into the treatment of a variety of bone defects [30,31].

Many advances have been achieved in the field of gene transfer; however, some problems such as poor efficiency and maintenance of cell characteristics remain. Transfection of hard-to-transfect cells, including primary neuron cells, primary blood cells, and stem cells through common methods such as the use of a liposome-based reagent or electroporation is problematic because of low transfection efficiency and low cell survival rates. 


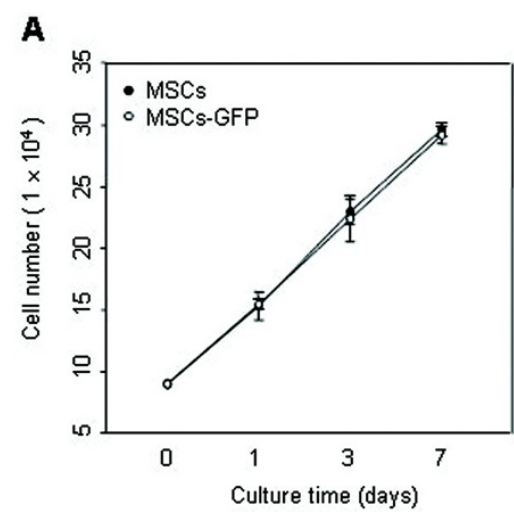

B
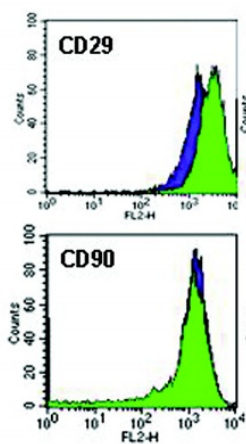
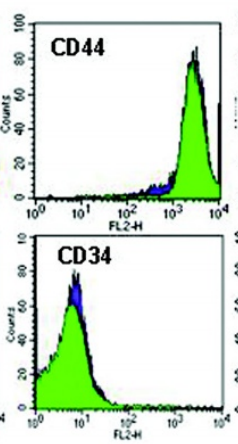
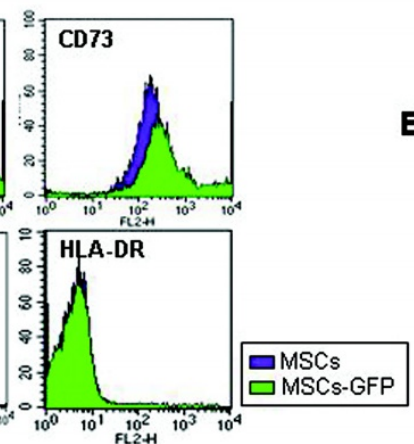

C

D

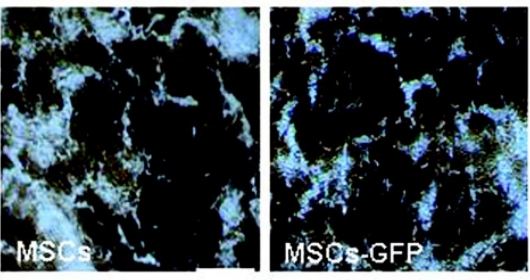

E
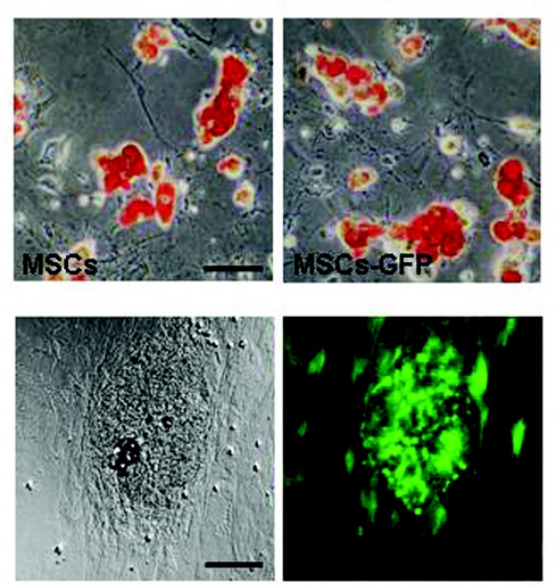

Figure 5 Multiple stem cell traits of EGFP-expressing hUCB-MSCs. (A) hUCB-MSCs were transfected with the $2 \mu$ g of pEGFP-N1 by microporation. After microporation, cells were cultured for additional $24 \mathrm{hr}$ and analyzed with the trypan blue exclusion assay from 1-7 days. (B) Cells were labeled with antibodies against the indicated antigens and they were then analyzed by flow cytometry. hUCB-MSCs were transfected with the $2 \mu \mathrm{g}$ of pEGFPN1. After transfection, cells were allowed to differentiate under the specific induction medium. The accumulation of calcium-containing mineral deposits in the extracellular matrix and lipidic vacuoles were revealed by the (C) von Kossa staining method and (D) Oil Red O solution after 3 weeks. (E) EGFP expression was maintained in hUCB-MSCs during the osteoinductive conditions. The data are expressed as the mean $\pm S E M ; n=3$. Scale bar (C) $=200 \mu \mathrm{m}$. Scale bar $(\mathrm{D}, \mathrm{E})=100 \mu \mathrm{m}$

Genetically modified cells should maintain the transgene wherever and whenever they are treated, as well as their cell characteristics. If MSCs can be successfully modified, more potential cells will be available for use in treatment. Therefore, technology and methodology that enables efficient gene transfer is needed.

In this study, we obtained successful transfection efficiency with only minimal cell damage by the microporation-based transfection with the EGFP reporter plasmid in hUCB-MSCs. Moreover, a plasmid expressing the gene of interest, BDNF, could be successfully transfected into hUCB-MSCs, and in vitro and in vivo experiments showed that BDNF expression remained fairly constant for the first 2 weeks (Figures 1, 2, 3, 4 and 7). These results show that gene delivery by microporaton is a safe and efficient means for prolonged transgene expression.

Recently, Wang et al. [32] demonstrated that microporation allows a highly efficient transfection of human adipose tissue-derived stem cells, without impairing their stem cell properties. Their results echo our data that transfection by microporation is an efficient way of obtaining successful gene delivery into hUCB-MSCs.

More importantly, microporation of BDNF gene into hUCB-MSCs presently promoted in vitro neural characteristics (Figure 8). MSCs can differentiate into neurons in vitro under special experimental conditions [33] and in vivo after transplantation into the brain and spinal cord [34,35]. In addition, transplanted MSCs promoted functional improvement in a spinal cord injury rat model [35]. Our previous results demonstrated that BDNF stimulates in vitro differentiation of MSCs into neural cells [27]. Furthermore, engineered MSCs with the BDNF gene effectively promote neural differentiation and also protect against injury in transected spinal cord or ischemia animal models $[27,28,35]$. Therefore, microporation-based successful genetic modification of hUCB-MSCs with BDNF makes them potential candidates for the treatment of neurological diseases.

Additionally, microporation-mediated transfection of pEGFP-N1 into hUCB-MSCs and EGFP overexpression did not affect the multiple potential of stem cells. This 
A

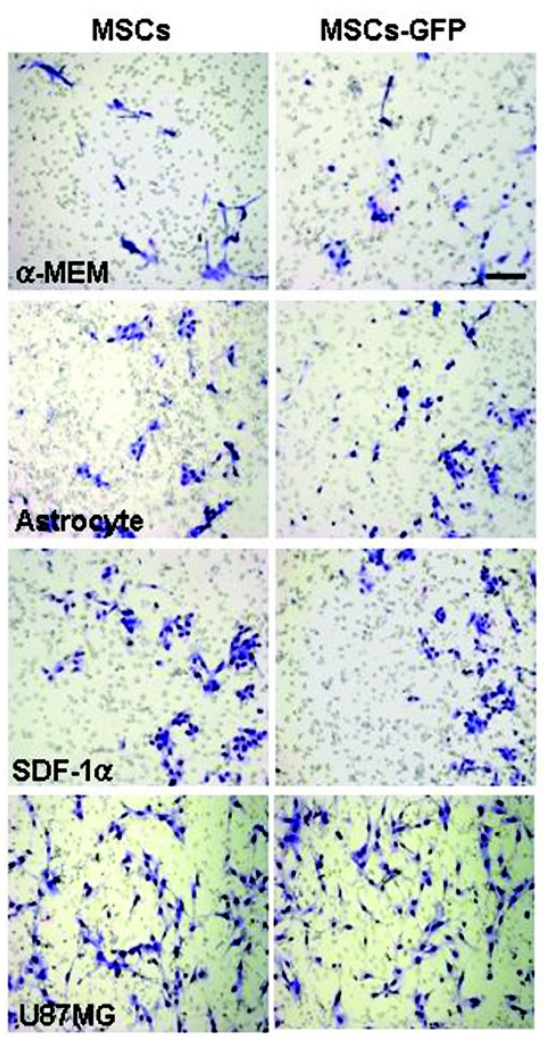

B

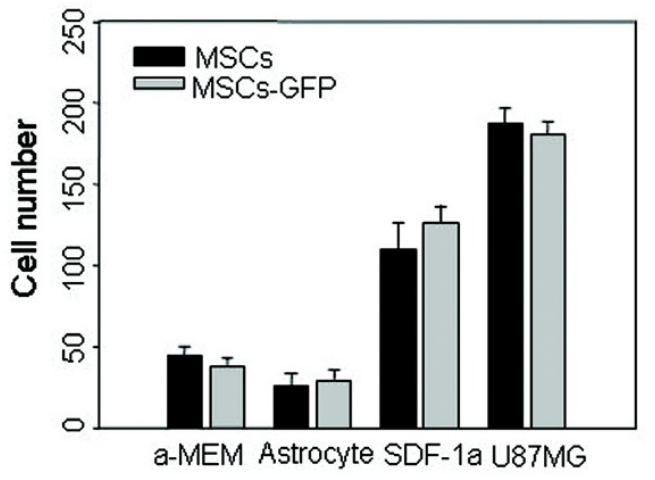

C
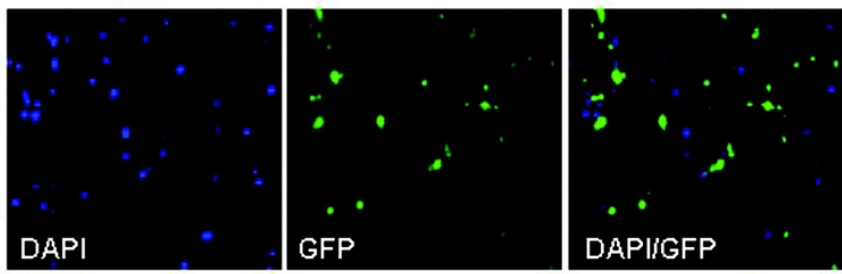

Figure 6 Migration capacity of EGFP-expressing hUCB-MSCs. (A) hUCB-MSCs were transfected with the $2 \mu \mathrm{g}$ of pEGFP-N1. After transfection, the migration response of hUCB-MSCs to conditioned medium from U-87MG cells or normal astrocytes was determined using a Transwell plate. Cell migration was compared and evaluated after staining by taking photographs and (B) counting cells that had migrated. Serum free medium was used as a negative control and SDF-1 was used as a positive control. The number of cells that had migrated to the lower side of the filter was counted in five or six different microscopic fields under a light microscope. (C) The expression of EGFP was observed in migrated hUCB-MSCs. Nuclei were counterstained with DAPI (blue). Experiments were performed in triplicate. Scale bar $=200 \mu \mathrm{m}$.

effect was demonstrated by cell phenotype characterization, proliferative activity, ability to differentiate into multiple lineages, and potent migration ability for malignant glioma cells (Figures 5 and 6). Maintenance of these multiple properties of modified stem cells is critical for improving their therapeutic potentials. Otherwise, stem cells may not be effective in a combination therapy of stem cells and therapeutic genes. Therefore, questions regarding the efficacy of gene transfection as well as safety and maintenance of multiple stem cell traits after gene modification need to be answered in greater detail for the effective utilization of stem cells in treating human diseases.

This study has established a reliable and efficient method for introducing a transgene into stem cells. Transfection by the microporation method led to $83 \%$ successful expression of transgene in hUCB-MSCs. In addition, multiple stem cell traits of hUCB-MSCs were unaffected by transfection. Successful genetic modification of stem cells requires achieving efficient DNA transfer into cells and maintaining their multiple cell characteristics. Therefore, transfection by the microporation is an efficient way of obtaining successful gene delivery into MSCs and gene delivery by microporation may enhance the feasibility of transgenic stem cell therapy.

\section{Conclusion}

Successful transfection efficiency was obtained with only minimal cell damage by the microporation-based transfection with the EGFP reporter plasmid in hUCB-MSCs without changing their multiple properties. Moreover, a plasmid expressing the gene of interest, BDNF, could be successfully transfected into hUCB-MSCs. More importantly, microporation of BDNF gene into hUCB-MSCs 
A
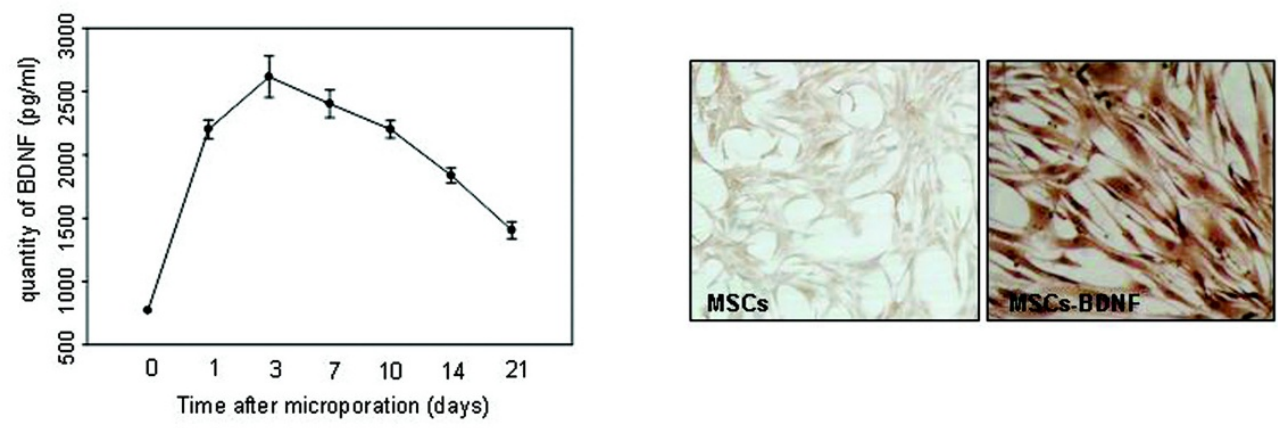

B
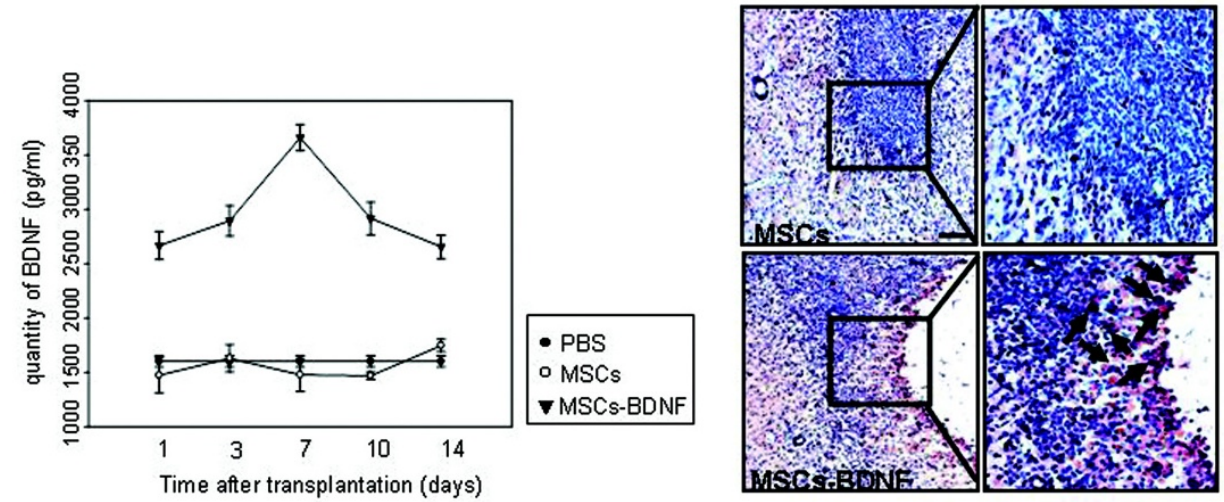

Figure 7 Time-course analysis of BDNF expressions in microporated hUCB-MSCs. (A, left panel) pEGFP-BDNF ( $2 \mu \mathrm{g})$ was transfected into hUCBMSCs by microporation. The concentration of secreted BDNF was determined using an ELISA assay under conditions of expanded cells (a-MEM containing 10\% FBS). (A, right panel) Cells were stained for BDNF 3 days after transfection. (B, left panel) For the quantification of BDNF levels and longevity of BDNF expression in vivo, brain tissues were homogenized at $1,3,7,10$, and 14 days ( $n=3$ /each group) after treatment and then assessed by ELISA. (B, right panel) Tissues were stained for BDNF 3 days after transplantation. Nuclei were counterstained with Hematoxylin (blue). The data are expressed as the mean $\pm \mathrm{SEM} ; \mathrm{n}=3$. Scale bar $=200 \mu \mathrm{m}$.

promoted their in vitro neural characteristics. Therefore, transfection by the microporation is an efficient way of obtaining successful gene delivery into MSCs and gene delivery by microporation may enhance the feasibility of transgenic stem cell therapy.

\section{Methods}

\section{Plasmid Construction}

An EGFP expression vector, pEGFP-N1, was purchased from Clontech BD Bioscience (Palo Alto, CA, USA). BDNF cDNA was obtained using the Marathon cDNA Amplification system (Clontech) and cloned in the TOPO TA Cloning vector (Invitrogen, Carlsbad, CA, USA) and the sequence was verified. Plasmid encoding human BDNF was generated by polymerase chain reaction (PCR) cloning into pEGFP-N1 using KpnI (5" primers) and NotI (3" primers) restriction sites, which was designated pEGFP-BDNF.

\section{hUCB-MSCs and Transfection}

Human UCB samples were obtained with consent from the mothers and were separated and maintained in accordance with techniques as previously described [36]. Cells were transfected with pEGFP-N1 or pEGFP-BDNF by liposome-based reagent, Gene Porter2 (Gene Therapy System, San Diego, CA, USA), established electroporators; Nucleofector" (Amaxa, Cologne, Germany), ECM 830 (BTX Harvard Apparatus, Holliston, MA, USA), MicroPulser $^{\text {TM }}$ (Bio-Rad, Huston, TX, USA) and a novel electroporator (MicroPorator ${ }^{\mathrm{Tm}}$, Digital Bio, Seoul, Korea).

For electroporation, cells were trypsinized and resuspended in PBS for electroporation with the ECM 830 or in resuspension buffer for electroporation with the MicroPulser $^{\mathrm{Tm}}$ and Nucleofector ${ }^{\circ}$. Then $2 \times 10^{5}$ cells were used for each electroporation. Briefly, $2 \mu \mathrm{g}$ DNA was mixed in PBS and electroporated as described previously $[23,24]$. For microporation, $2 \mu \mathrm{g}$ DNA was mixed with 10 
A
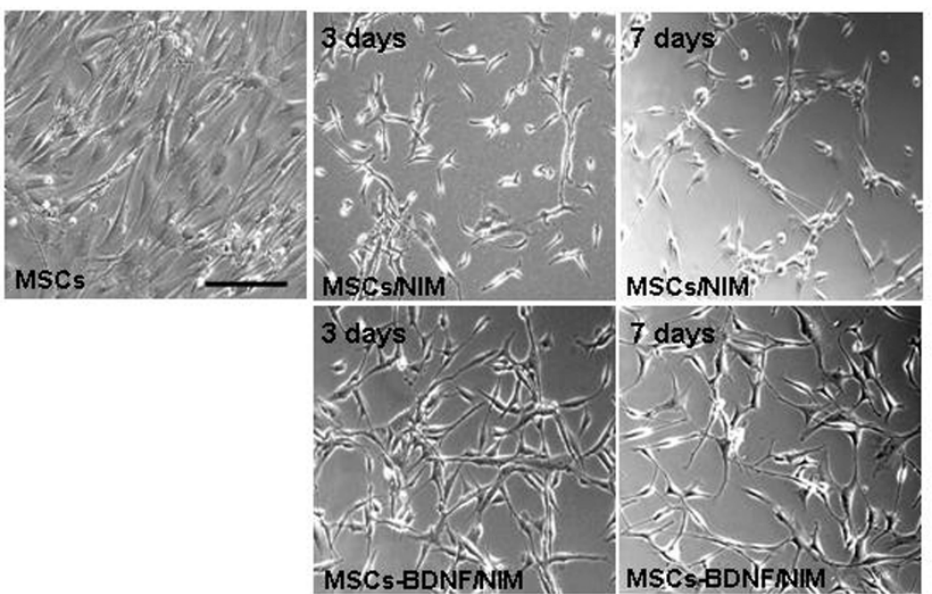

B

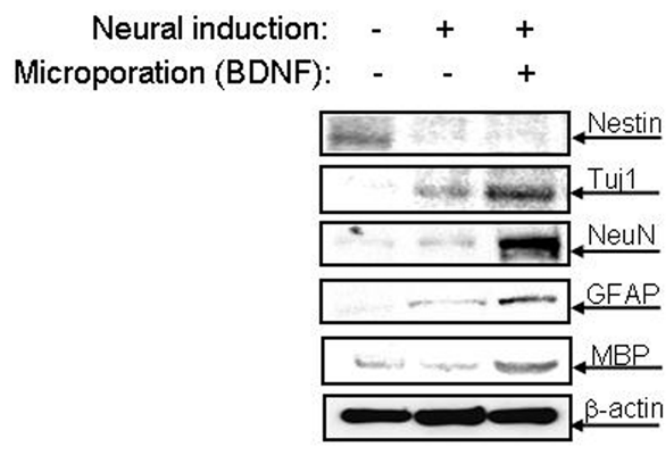

C

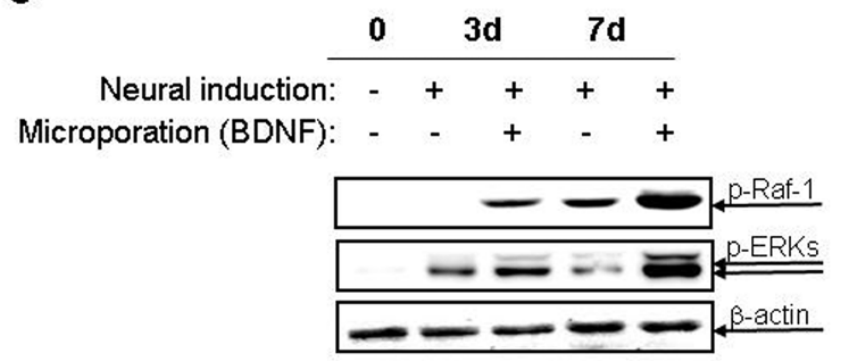

Figure 8 Neurogenic characteristics of BDNF-expressing hUCB-MSCs. (A) phase-contrast microscopy analysis of hUCB-MSCs after 3 and 7 days incubation in NIM, with or without microporation-based pEGFP-BDNF transfection $(2 \mu \mathrm{g})$. (B) Western blotting of hUCB-MSCs before and 3 days of incubation in NIM with or without pEGFP-BDNF transfection. The cell lysates were then subjected to SDS-polyacrylamide gel electrophoresis and Western blot analysis with anti-nestin, anti-Tuj-1, anti-NeuN, anti-GFAP, and anti-MBP antibodies. (C) Western blotting of hUCB-MSCs before and 3 and 7 days of incubation in NIM with or without pEGFP-BDNF transfection. The cell lysates were then subjected to SDS-polyacrylamide gel electrophoresis and Western blot analysis with anti-phospho ERK,-phospho -Raf-1 antibodies. Equal amounts of total protein were confirmed by using an anti- $\beta$-actin antibody. The results shown here are from one of two independent experiments. Scale bar $=100 \mu \mathrm{m}$

$\mu \mathrm{l}$ resuspension buffer. Then, $2 \times 10^{5}$ cells were electroporated with a pre-optimized square pulse condition (1600 $\mathrm{V}, 20 \mathrm{~ms}, 1$ pulse). Electroporated cells were incubated in $500 \mu \mathrm{l} \alpha$-MEM (Invitrogen) supplemented with 10\% FBS (Invitrogen) without antibiotics for $24 \mathrm{hr}$, and then the medium was replaced with a medium containing antibiotics. To assay transient and sustained expression, the hUCB-MSCs were isolated by incubation in $0.25 \%$ trypsin and $1 \mathrm{mM}$ EDTA (Invitrogen) and assayed by a FACSCalibur flow cytometer (Becton Dickinson, Franklin Lakes, NJ, USA) $24 \mathrm{hr}$ after transfection for EGFPexpressing cells. The results were analyzed by CellQuest software (Becton Dickinson).

\section{Assessment of Cell Viability}

hUCB-MSCs were transfected with pEGFP-N1 by various transfection. Medium was removed $24 \mathrm{hr}$ after transfection, and replaced with medium containing antibiotics. Cells were analyzed for viability by CellTiter $96^{\circ}$ aqueous non-radioactive cell proliferation assay (Promega, Madison, WI, USA) at different time points after transfection. The assay tests cellular viability and mitochondrial function. Briefly, after transfection, MTS solution was added to each culture plate and plates were incubated at $37^{\circ} \mathrm{C}$ for $3 \mathrm{hr}$. The absorbance of the formazan product, which is considered to be directly proportional to the number of living cells in the culture, was measured at $490 \mathrm{~nm}$ using an ELISA plate reader. 


\section{Animals and Cell Transplantation}

Adult male SD rats (6-8 weeks old; Charles River Laboratories, Wilmington, MA, USA) were used in accordance with institutional guidelines under the approved protocols. Cells were transfected with pEGFP-BDNF and maintained further for $48 \mathrm{hr}$ in $\alpha$-MEM containing 10\% FBS. For the intracranial xenografts of hUCB-MSCs, animals were anesthetized with an intraperitoneal injection of ketamine/xylazine and $3 \times 10^{5}$ cells were stereotactically transplanted into the right frontal lobe $(2.6 \mathrm{~mm}$ lateral and $1.2 \mathrm{~mm}$ anterior to bregma, at $5.0 \mathrm{~mm}$ depth from the skull base) via a Hamilton syringe (Hamilton Company, Reno, NV, USA) using a microinfusion pump (Harvard Apparatus, Holliston, MA, USA).

\section{ELISA for Expressed BDNF}

To investigate the persistence of transgene expression in vitro, hUCB-MSCs were transfected with pEGFP-BDNF by MicroPorator ${ }^{\mathrm{rm}}$. Culture supernatants were harvested, fresh medium ( $\alpha$-MEM containing 10\% FBS) was added, and secreted BDNF was assessed at various time intervals. To investigate the persistence of BDNF expression in vivo, brain tissues ( $2 \mathrm{~mm}$ segment centered on the injection site) were harvested and lysed in a RIPA buffer at 1 , $3,7,10$, and 14 days after treatment of cells. BDNF protein secreted into the culture supernatants or brain tissues was analyzed by ELISA assay kits (R\&D Systems, Minneapolis, MN, USA).

\section{Immunophenotyping of hUCB-MSCs}

To analyze the cell surface expression of typical marker proteins in hUCB-MSCs, cells were labeled with the following anti-human antibodies: CD29-PE, CD34-PE, CD44-PE, CD73-PE, CD90-PE, and HLA-DR-PE (BD Biosciences). Ten thousand cells were measured using a FACSCalibur flow cytometer (Becton Dickinson) and the results were analyzed with CellQuest software (Becton Dickinson).

\section{Multidifferentiation of Cultured hUCB-MSCs}

Differentiation to adipogenic and osteogenic lineages was induced according to previously described procedures [2]. After 2-3 weeks of culture, differentiated cells were fixed with $3 \%$ formaldehyde. Adipocytes were detected by staining the lipid droplets in the cell using $0.3 \%$ Oil red-O staining for $10 \mathrm{~min}$. Osteocytes were detected by calcium phosphate deposits after von Kossa staining. In brief, cells were fixed with ethanol and stained with $5 \%$ silver nitrate for $1 \mathrm{hr}$. After rinsing with distilled water, the cells were incubated in 5\% sodium thiosulfate for 2 min to allow precipitation of insoluble black silver granules around calcium phosphate.

\section{Immunocytochemistry}

After transfection, cells were fixed with $4 \%$ paraformaldehyde for $10 \mathrm{~min}$ and processed for immunocytochemistry to identify neural marker-positive cells. Nonspecific antibody reactions were blocked with $5 \%$ horse serum for 1 $\mathrm{hr}$ at room temperature (RT). Next, the fixed cells were incubated overnight at $4^{\circ} \mathrm{C}$ with primary antibodies directed against BDNF (Chemicon, Temecula, CA, USA). After three washes, cells were incubated with biotinylated secondary antibodies (Vector Laboratories, Burlingame, CA, USA) for $1 \mathrm{hr}$ at RT, followed by $1 \mathrm{hr}$ of incubation in avidin-biotinylated peroxidase complex (Vector Elite Kit, Vector Laboratories) at RT. Diaminobenzidine $(0.05 \%)$ with nickel chloride $(0.04 \%)$ was used as the chromagen, and reactions were sustained for 1-6 $\mathrm{min}$ at RT. The fixed cells were then coverslipped with Fluoromount G (Southern Biotechnology Associates, Birmingham, AL, UK).

\section{Immunohistochemistry}

Rat brains were perfused with PBS followed by $4 \%$ paraformaldehyde under deep anesthesia at 3 days after transplantation of hUCB-MSCs or transfected hUCB-MSCs. The excised brains were postfixed overnight and then equilibrated in PBS containing 30\% sucrose for 2 days. Fixed brains were embedded, snap frozen in liquid nitrogen, and stored at $-70^{\circ} \mathrm{C}$ until use. Tissues were cryosectioned $(16 \mu \mathrm{m})$ and then stained with primary antibodies for anti-BDNF (Chemicon). After three washes, tissues were incubated with biotinylated secondary antibodies (Vector Laboratories) for $1 \mathrm{hr}$ at RT, followed by $1 \mathrm{hr}$ of incubation in avidin-biotinylated peroxidase complex (Vector Elite Kit, Vector Laboratories) at RT. Vector NovaRED (Vector Laboratories) was used for visualizing the immunoreaction products, and reactions were sustained for 1-6 min at RT. Nuclei were counterstained with Hematoxylin.

\section{In vitro Migration Assay}

The migratory ability of hUCB-MSCs was determined using Transwell plates (Corning Costar, Cambridge, MA, USA) that were $6.5 \mathrm{~mm}$ in diameter with $8 \mu \mathrm{m}$ pore filters. $1 \times 10^{6}$ of cells were incubated in $5 \mathrm{ml}$ serum free MEM for U-87MG or DMEM for astrocytes for $48 \mathrm{hr}$, and the resulting conditioned media were used as chemoattractants. hUCB-MSCs or hUCB-MSCs transfected with pEGFP-N1 $\left(2 \times 10^{4}\right)$ were suspended in $100 \mu \mathrm{l}$ serum free medium (SFM) containing $0.1 \%$ bovine serum albumin (Sigma) and seeded into the upper well, and 600 $\mu \mathrm{l}$ of conditioned medium (CM) was placed in the lower well of the Transwell plate. Following incubation for $5 \mathrm{hr}$ at $37^{\circ} \mathrm{C}$, cells that had not migrated from the upper side of the filters were scraped off with a cotton swab, and filters were stained with the three-step stain set (Diff-Quik; Sys- 
mex, Kobe, Japan). The number of cells that had migrated to the lower side of the filter was counted under a light microscope $(\times 200)$. Experiments were performed in triplicate.

\section{Neural Induction Procedure}

Neural induction was performed in accordance with the procedure described by Lim et al. [27]. In brief, cells were plated in $\alpha$-MEM containing $10 \%$ FBS at 4000 cells $/ \mathrm{cm}^{2}$. Twenty-four hours before induction, the medium was replaced with a preinduction medium composed of $\alpha$ MEM, $10 \% \mathrm{FBS}$ and $10 \mathrm{ng} / \mathrm{ml}$ of basic fibroblast growth factor (bFGF, Invitrogen). The cells were induced by replacing the pretreatment medium with neural induction medium (NIM), consisting of $100 \mu \mathrm{M}$ butylated hydroxyanisole (Sigma), 2\% dimethylsulfoxide (Sigma), $25 \mathrm{mM} \mathrm{KCl}, 5 \mathrm{U} / \mathrm{ml}$ heparin (Sigma), $20 \mathrm{ng} / \mathrm{ml} \mathrm{bFGF,} 5$ $\mu \mathrm{g} / \mathrm{ml}$ insulin (Sigma), $100 \mu \mathrm{g} / \mathrm{ml}$ transferring (Sigma), 20 nM progesterone (Sigma), $100 \mu \mathrm{M}$ putrescine (Sigma), 30 $\mathrm{nM}$ sodium selenite (Sigma), and $0.5 \mu \mathrm{M}$ all-trans-retinoic acid (Sigma). After induction, the cells were maintained in NIM for up to 7 days.

\section{Western Blot Analysis}

Antibodies were obtained from commercial sources: BDNF, Nestin, $\beta$ III tubulin, NeuN, and MBP antibodies from Chemicon; GFAP antibody from Dako; p-ERKs, and p-Raf-1 antibodies from New England Biolabs (Ipswich, $\mathrm{MA})$. For the Western blot analysis, the cells were rinsed with PBS and subsequently lysed for $30 \mathrm{~min}$ on ice in RIPA-B buffer $(0.5 \%$ Nonidet P-40, $20 \mathrm{mM}$ Tris, $\mathrm{pH}$ 8.0, $50 \mathrm{mM} \mathrm{NaCl}, 50 \mathrm{mM} \mathrm{NaF}, 100 \mu \mathrm{M} \mathrm{Na} 3 \mathrm{VO} 4,1 \mathrm{mM}$ DTT, and $50 \mu \mathrm{g} / \mathrm{ml} \mathrm{PMSF).} \mathrm{The} \mathrm{insoluble} \mathrm{material} \mathrm{was}$ removed by centrifugation at 12,000 rpm for $20 \mathrm{~min}$ at 48 C. Next, the supernatant was subjected to SDS-PAGE, and the Western blot analysis was then performed. The blots were blocked in PBS with 5\% skim milk and 0.05\% Tween 20, incubated with the appropriate antibodies and subsequently incubated with the secondary antibodies conjugated with horseradish peroxidase. Next, the blots were assayed using an enhanced chemiluminescence detection system (Amersham Biosciences, Piscataway NJ, USA).

\section{Authors' contributions \\ JYL conceived the method, generated the experimental data, and wrote the first draft of the manuscript. SSJ participated in the evaluation of the data. Cell transplantation and in vitro migration experiments were performed by $\mathrm{CHJ}$ and SMK with help and advice from JAJ and CHR. Microporation experiments were performed by $\mathrm{JHO}$ and SHP. Immunostaining experiments were performed JGA and SAP. hUCB-MSCs were prepared by JWC and WIO. All authors read and approved the final manuscript.}

\section{Acknowledgements}

This study was supported by a grant of the Korea Health 21 R\&D Project, Ministry of Health and Welfare, Republic of Korea (0405-DB01-0104-0006) and by a grant of the, National R\&D Program for Cancer Control, Republic of Korea (0820040), and by Basic Science Research program through the National Research Foundation of Korea (NRF) funded by the Ministry of Education, Science and Technology (2009-0067091), Republic of Korea.

\section{Author Details}

'Department of Biomedical Science, College of Medicine, The Catholic University of Korea, Seoul, Korea, 2 Department of Neurosurgery, Seoul St. Mary's Hospital, The Catholic University of Korea, Seoul, Korea, ${ }^{3}$ Department of Neurosurgery, St. Paul's Hospital, The Catholic University of Korea, Seoul, Korea and ${ }^{4}$ Medipost Biomedical Research Institute, MEDIPOST Co., Ltd., Seoul, Korea

Received: 6 October 2009 Accepted: 13 May 2010

Published: 13 May 2010

\section{References}

1. Goodwin HS, Bicknese AR, Chien SN, Boqucki BD, Quinn CO, Wall DA: Multilineage differentiation activity by cells isolated from umbilical cord blood: expression of bone, fat, and neural markers. Biol Blood Marrow Transplant 2001, 7:581-588

2. Pittenger MF, Mackay AM, Beck SC, Jaiswal PK, Douglas R, Mosca JD, Moorman MA, Simonetti DW, Craig S, Marshak DR: Multilineage potential of adult human mesenchymal stem cells. Science 1999, 284:143-147.

3. Caplan Al: Mesenchymal stem cells. J Orthop Res 1991, 9:641-650.

4. Parr AM, Tator $\mathrm{CH}$, Keating A: Bone marrow-derived mesenchymal stromal cells for the repair of central nervous system injury. Bone Marrow Transplant 2007, 40:609-619.

5. Li Y, Chen J, Chen XG, Wang L, Gautam SC, Xu YX, Katakowski M, Zhang LJ, Lu M, Janakiraman N, Chopp M: Human marrow stromal cell therapy for stroke in rat: neurotrophins and functional recovery. Neurology 2002, 59:514-523.

6. Studeny M, Marini FC, Dembinski JL, Zompetta C, Cabreira-Hansen M, Bekele BN, Champin RE, Andreeff M: Mesenchymal stem cells: potential precursors for tumor stroma and targeted delivery vehicles for anticancer agents. J Natl Can Inst 2004, 96:1593-1603.

7. Studeny M, Marini FC, Champlin RE, Zompetta C, Fidler IJ, Andreeff M: Bone marrow derived mesenchymal stem cells as vehicles for interferon-b delivery into tumors. Cancer Res 2002, 62:3603-3608.

8. Le Blanc K, Tammik L, Sundberg B, Haynesworth SE, Ringden O: Mesenchymal stem cells inhibit and stimulate mixed lymphocyte cultures and mitogenic responses independently of the major histocompatibility complex. Scand J Immunol 2003, 57:11-20.

9. Nakamizo A, Marini F, Amano T, Khan A, Studeny M, Gumin J, Chen J, Hentschel S, Vecil G, Dembinski J, Andreeff M, Lang FF: Human bone marrow-derived mesenchymal stem cells in the treatment of gliomas. Cancer Res 2005, 65:3307-3318.

10. Consiglio A, Gritti A, Dolcetta D, Follenzi A, Bordiqnon C, Gage FH, Vesconvi AL, Naldini L: Robust in vivo gene transfer into adult mammalian neural stem cells by lentiviral vectors. Proc Natl Acad SCi USA 2004, 101:14835-14840.

11. Park F, Ohashi K, Chiu W, Naldini L, Kay MA: Efficient lentiviral transduction of liver requires cell cycling in vivo. Nat Genet 2000, 24:49-52.

12. Naldini L, Blomer U, Gage FH, Trono D, Verma IM: Efficient transfer, integration, and sustained long-term expression of the transgene in adult rat brains injected with a lentiviral vector. Proc Natl Acad Sci USA 1996, 93:11382-11388.

13. Olmsted-Davis EA, Gugala Z, Gannon FH, Yotnda P, McAlhany RE, Lindsey RW, Davis AR: Use of a chimeric adenovirus vector enhances BMP2 production and bone formation. Hum Gene Ther 2002, 13:1337-1347.

14. Kushibiki T, Tabata Y: A new gene delivery system based on controlled release technology. Current Drug Delivery 2004, 1:153-163.

15. Hall KM, Horvath TL, Abonour R, Cornetta K, Srour EF: Decreased homing of retrovirally transduced human bone marrow CD34+ cells in the NOD/SCID mouse model. Exp Hemato/ 2006, 34:433-442.

16. Nishikawa $M$, Huang L: Nonviral vectors in the new millennium:delivery barriers in gene transfer. Hum Gene Ther 2001, 12:861-870.

17. Almofti MR, Harashima H, Shinohara Y, Almofti A, Li W, Kiwada H: Lipoplex size determines lipofection efficiency with or without serum. Mol Membr Biol 2003, 20:35-43. 
18. Kubiniec RT, Liang H, Hui SW: Effect of pulse length and pulse strength on transfection by electroporation. BioTechniques 1990, 8:16-20.

19. Watanabe SY, Albsoul-Younes AM, Kawano T, Itoh H, Kaziro Y, Nakajima S, Nakajima Y: Calcium phosphate-mediated transfection of primary cultured brain neurons using GFP expression as a marker: application for single neuron electrophysiology. Neurosci Res 1999, 33:71-78.

20. Teissie J, Golzio M, Rols MP: Mechanisms of cell membrane electropermeabilization: a minireview of our present knowledge. Biochim Biophys Acta 2005, 1724:270-280

21. Mir LM: Therapeutic perspectives of in vivo cell electropermeabilization. Bioelectrochemistry 2001, 53:1-10.

22. Mir LM, Bureau MF, Gehl J, Rangara R, Rouy D, Caillaud JM, Delaere $P$, Branellec D, Schwartz B, Scherman D: High-efficiency gene transfer into skeletal muscle mediated by electric pulses. Proc Natl Acad Sci USA 1999, 96:4262-4267.

23. Colleoni S, Donofrio G, Lagutina I, Duchi R, Galli C, Lazzari G: Establishment, differentiation, electroporation, viral transduction, and nuclear transfer of bovine and porcine mesenchymal stem cells. Cloning Stem Cells 2005, 7:154-165.

24. Peister A, Mellad JA, Wang M, Tucker HA, Prockop DJ: Stable transfection of MSCs by electroporation. Gene Therapy 2004, 11:224-228.

25. Huang EJ, Reichardt LF: Trk receptors: roles in neuronal signal transduction. Annu Rev Biochem 2003, 72:609-642.

26. Ahmed S, Reynolds BA, Weiss S: BDNF enhances the differentiation but not the survival of CNS stem cell-derived neuronal precursors. $J$ Neurosci 1995, 15:5765-5778.

27. Lim JY, Park SI, Kim SM, Oh JH, Jeong CH, Jun JA, Lee KS, Oh W, Lee JK, Jeun SS: Brain-derived neurotrophic factor stimulates the neural differentiation of human umbilical cord blood-derived mesenchymal stem cells and survival. J Neurosci Res 2008, 86:2168-2178.

28. Lu P, Jones LL, Tuszynski MH: BDNF-expressing marrow stromal cells support extensive axonal growth at sites of spinal cord injury. Exp Neurol 2005, 191:344-360.

29. Kurozumi K, Nakamura K, Tamiya T, Kawano Y, Kobune M, Hirai S, Uchida H, Sasaki K, Ito Y, Kato K, Honmou O, Houkin K, Date I, Hamada H: BDNF gene-modified mesenchymal stem cells promote functional recovery and reduce infarct size in the rat middle cerebral artery occlusion model. Mol Ther 2004, 9:189-197.

30. Tsuda H, Wada T, Ito Y, Uchida H, Dehari H, Nakamura K, Sasaki K, Kobune M, Yamashita T, Hamada H: Efficient BMP2 gene transfer and bone formation of mesenchymal stem cells by a fiber mutant adenoviral vector. Mol Ther 2003, 7:354-365.

31. Gazit D, Turgeman G, Kelley P, Wang E, Jalenak M, Zilberman Y, Moutsatsos I: Engineered pluripotent mesenchymal cells integrate and differentiate in regenerating bone: a novel cell-mediated gene therapy. J Gene Med 1999, 1:121-133.

32. Wang YH, Ho ML, Chang JK, Chu HC, Lai SC, Wang GJ: Microporation is a valuable transfection method for gene expression in human adipose tissue-derived stem cells. Mol Ther 2009, 17:302-308.

33. Jeong JA, Gang EJ, Hong SH, Hwang SH, Kim SW, Yang IH, Ahn C, Han H, Kim H: Rapid neural differentiation of human cord blood-derived mesenchymal stem cells. Neuroreport 2004, 15:1731-1734.

34. Zigova T, Song S, Willing AE, Hudson JE, Newman MB, Saporta S, SanchezRamos J, Sanberg PR: Human umbilical cord blood cells express neural antigens after transplantation into the developing rat brain. Cell Transplant 2002, 11:265-274

35. Kuh SU, Cho YE, Yoon DH, Kim KN, Ha Y: Functional recovery after human umbilical cord blood cells transplantation with brain-derived neutrophic factor into the spinal cord injured rat. Acta Neurochir 2005, 147:985-992.

36. Yang $\mathrm{SE}$, Ha CW, Jung M, Jin HJ, Lee M, Song H, Choi S, Oh W, Yang YS: Mesenchymal stem/progenitor cells developed in cultures from UC blood. Cytotherapy 2004, 6:476-486.

doi: $10.1186 / 1472-6750-10-38$

Cite this article as: Lim et al., Microporation is a valuable transfection method for efficient gene delivery into human umbilical cord blood-derived mesenchymal stem cells BMC Biotechnology 2010, 10:38

\section{Submit your next manuscript to BioMed Central} and take full advantage of:

- Convenient online submission

- Thorough peer review

- No space constraints or color figure charges

- Immediate publication on acceptance

- Inclusion in PubMed, CAS, Scopus and Google Scholar

- Research which is freely available for redistribution 